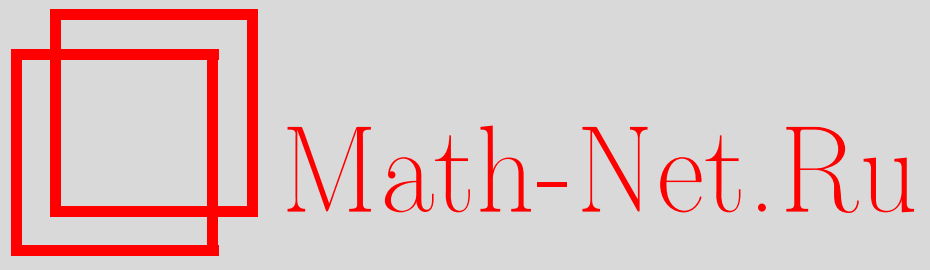

А. Г. Кукуш, Ю. С. Мишура, Асимптотические свойства оценки интенсивности неоднородного пуассоновского процесса в комбинированной модели, Теория вероятн. и ее примен., 1999, том 44, выпуск 2, 351-372

DOI: https://doi.org/10.4213/tvp770

Использование Общероссийского математического портала Math-Net.Ru подразумевает, что вы прочитали и согласны с пользовательским соглашением http: //www . mathnet.ru/rus/agreement

Параметры загрузки:

IP: 54.224 .135 .184

26 апреля 2023 г., 16:03:58

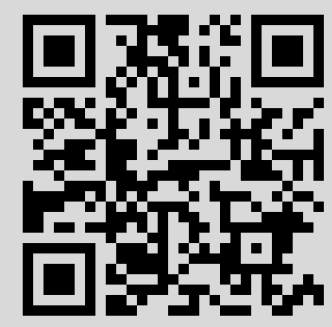




\section{АСИМПТОТИЧЕСКИЕ СВОЙСТВА ОЦЕНКИ ИНТЕНСИВНОСТИ НЕОДНОРОДНОГО ПУАССОНОВСКОГО ПРОЦЕССА В КОМБИНИРОВАННОЙ МОДЕЛИ}

Рассматривается случайный процесс, имеющий снос, диффузию и пуассоновскую компоненту, причем последняя является неоднородным процессом с неизвестной интенсивностью $\lambda=\lambda(t)$, принадлежащей компакту из некоторого соболевского пространства. По наблюдениям процесса на отрезке $[0, T]$ строится оценка максимального правдоподобия (ОМП) функции $\lambda$. Изучаются условия ее состоятельности и асимптотической нормальности функционалов от оценки. Приведено сравнение ОМП, построенных по наблюдениям за процессом в целом и за отдельными его компонентами.

Ключевые слова и фразы: неоднородный пуассоновский процесс, интенсивность, снос, диффузия, оценка максимального правдоподобия, состоятельность, асимптотическая нормальность, соболевское пространство.

1. Введение. Рассматриваемая схема состоит в следующем. На неотрицательной полуоси наблюдается случайный прощесс с независимыми приращениями, состояпий из трех компонент: сноса, диффузии и неоднородного пуассоновского процесса с неизвестной интенсивностью $\lambda=\lambda(t), t \geqslant 0$. По наблюдениям за траекториями процесса строится оценка максимального правдоподобия для $\lambda(t)$. В связи с рассматриваемой схемой упомянем следуюшие работы.

Непараметрические оценки коэффициента сноса диффузионного процесса исследовались в монографиях А. Я. Дороговцева [1], а также И. ШІ. Ибрамхалилова и А. В. Скорохода [2]. В статье А. Я. Дороговцева и А. Г. Кукуша [3] изучалась непараметрическая оценка интенсивности неоднородного процесса Пуассона. Особенность настоящей работы состоит в том, что оцениваемая функция интенсивности нелинейно входит в коэффициент сноса наблюдаемого процесса.

*Киевский университет им. Т. Шевченко, механико-математический факультет, ул. Владимирская, 64, 252601 Киев, Украина; e-mail: myus@mechmat.univ.kiev.ua. 
Обозначим $\mathbf{R}^{+}:=[0, \infty)$. Пусть на стохастическом базисе $\left\{\Omega, \mathscr{F}, \mathscr{F}_{t}, t \geqslant 0, \mathbf{P}\right\}$ с полной вероятностной мерой $\mathbf{P}$ на отрезке $[0, T]$ наблюдается траектория случайного процесса $\left\{\xi_{t}, t \geqslant 0\right\}$. Процесс имеет представление

$$
\xi_{t}=\xi_{t}\left(\lambda_{0}\right):=\xi_{0}+\int_{0}^{t} a\left(s, \lambda_{0}(s)\right) d s+\int_{0}^{t} b(s) d w_{s}+N_{t}\left(\lambda_{0}\right),
$$

где $a=a(s, x): \mathbf{R}^{+} \times \mathbf{R}^{+} \longrightarrow \mathbf{R}, b=b(s): \mathbf{R}^{+} \rightarrow \mathbf{R}$ - известные неслучайные измеримые функции, $\left\{w_{t}, \mathscr{F}_{t}, t \geqslant 0\right\}$ - винеровский процесс, $\left\{N_{t}\left(\lambda_{0}\right), \mathscr{F}_{t}, t \geqslant 0\right\}-$ неоднородный пуассоновский процесс с неизвестной интенсивностью $\lambda_{0}=\lambda_{0}(t)$, причем процессы $\left\{w_{t}\right\},\left\{N_{t}\left(\lambda_{0}\right)\right\}$ и случайная величина $\xi_{0}$ независимы в совокупности. Предполагается, что неслучайная функция $\lambda_{0}: \mathbf{R}^{+} \rightarrow \mathbf{R}^{+}, \lambda_{0} \in L_{\text {loc }}^{1}$. Траектории процесса $\left\{N_{t}\right\}$ c вероятностью 1 принадлежат пространству $X$ ступенчатых функций $x$, непрерывных справа и таких, что $x_{0}=0, \Delta x_{t}=0$ или 1 . Сужение $X$ на отрезок $[0, T]$ обозначим через $X_{T}$. В дальнейшем выражения типа $a(s, \lambda(s))$, стояшие под знаком интеграла, будем записывать: кратко $a(s, \lambda)$.

С вероятностной точки зрения, схема наблюдений (1) эквивалентна одновременному наблюдению двух независимых случайных процессов $\left\{N_{t}\left(\lambda_{0}\right), \mathscr{F}_{t}, t \geqslant 0\right\}$ и $\left\{\eta_{t}\left(\lambda_{0}\right), \mathscr{F}_{t}, t \geqslant 0\right\}$. Здесь

$$
\eta_{t}\left(\lambda_{0}\right):=\xi_{0}+\int_{0}^{t} a\left(s, \lambda_{0}\right) d s+\int_{0}^{t} b(s) d w_{s}
$$

пусть $Y_{T}:=C[0, T]-$ пространство, в котором лежат траектории этого процесса на отрезке $[0, T]$. Паре процессов $\left\{N_{t}\left(\lambda_{0}\right), \eta_{t}\left(\lambda_{0}\right), t \in[0, T]\right\}$ соответствует некоторая мера $\mu_{T}\left(\lambda_{0}\right)$ в пространстве $X_{T} \times Y_{T}$. Пусть $\lambda_{1} \in L_{\text {loc }}^{1}$ - другая интенсивность пуассоновской компоненты. Согласно $[4, \S 47$, с. 264], [5, § 8, с. 343, теорема 14] при выполнении условий:

$$
\begin{aligned}
& \text { (a) } \int_{0}^{T} \frac{\lambda_{1}(s)}{\lambda_{0}(s)} d s<\infty \\
& \text { (b) } \int_{0}^{T} b^{-2}(s)\left|a\left(s, \lambda_{1}\right)-a\left(s, \lambda_{0}\right)\right|^{2} d s<\infty
\end{aligned}
$$

мера $\mu_{T}\left(\lambda_{1}\right) \ll \mu_{T}\left(\lambda_{0}\right)$, и отношение правдоподобия имеет вид:

$$
\begin{aligned}
\frac{d \mu_{T}\left(\lambda_{1}\right)}{d \mu_{T}\left(\lambda_{0}\right)} & \left(N_{T}\left(\lambda_{0}\right), \eta_{t}\left(\lambda_{0}\right)\right) \\
=\exp & \left\{\int_{0}^{T} b^{-1}(s)\left(a\left(s, \lambda_{1}\right)-a\left(s, \lambda_{0}\right)\right) d w_{s}\right. \\
& \quad-\frac{1}{2} \int_{0}^{T} b^{-2}(s)\left|a\left(s, \lambda_{1}\right)-a\left(s, \lambda_{0}\right)\right|^{2} d s \\
& \left.\quad-\int_{0}^{T}\left(\lambda_{1}(s)-\lambda_{0}(s)\right) d s+\int_{0}^{T} \ln \frac{\lambda_{1}(s)}{\lambda_{0}(s)} d N_{s}\left(\lambda_{0}\right)\right\} .
\end{aligned}
$$


2. Построение оценки. Теперь оценка максимального правдоподобия (ОМII) функции $\lambda_{0}$ будет строиться путем максимизации правой части (2). Введем необходимые пространства. При $r>1$ обозначим через $\bar{W}_{r}^{1}[0, \tau]$ соболевское пространство функций $\lambda$, заданных на $[0, \tau]$, имеющих обобщенную производную $\lambda^{\prime} \in L_{\tau}[0, \tau]$ (определение обобщенной производной см. в $[6$, с. 441]) и таких, что $\lambda(0)=\lambda(\tau)$;

$$
\|\lambda\|_{\bar{W}_{r}^{1}}:=\left(\int_{0}^{\tau}|\lambda(t)|^{r} d t+\int_{0}^{\tau}\left|\lambda^{\prime}(t)\right|^{r} d t\right)^{1 / \tau} .
$$

3 а м е ч а н и е 1. Справедливы следуюшие теоремы вложения Соболева [6, с. 439,444$]$.

a) При $r>1$ пространство $\bar{W}_{r}^{1}[0, \tau]$ компактно вложено в пространство $C[0, \tau]$.

b) При $1<r<s$ пространство $\bar{W}_{s}^{1}[0, \tau]$ компактно вложено в пространство $\bar{W}_{\tau}^{1}[0, \tau]$.

Рассмотрим следующие предположения о функции интенсивности.

(c) Функция $\lambda_{0}$ периодична с известным периодом $\tau>0$.

(d) Сужение $\lambda_{0}$ на $[0, \tau]$ принадлежит $K$, где $K-$ компакт в пространстве $\bar{W}_{2}^{1}[0, \tau]$, причем все функции из $K$ неотрицательны.

Введем несколько обозначений, связанных с компактом $K$ :

$$
\bar{K}:=\sup _{\lambda \in K}\|\lambda\|_{\bar{W}_{2}^{1}}, \quad \lambda_{*}(s):=\min _{\lambda \in K} \lambda(s), \lambda^{*}(s):=\max _{\lambda \in K} \lambda(s) .
$$

Пусть также $\lambda_{*}$ и $\lambda^{*}$ суть, соответственно, минимум функции $\lambda_{*}(s)$ и максимум функции $\lambda^{*}(s)$ на $[0, \tau]$. Далее не будем делать различия между функциями из $K$ и их $\tau$-периодическими продолжениями на $\mathbf{R}^{+}$. Функции $\lambda_{*}(s)$ и $\lambda^{*}(s)$ будем считать продолженными с периодом $\tau$ на $\mathbf{R}^{+}$.

$$
\text { (e) } \int_{0}^{\tau} \frac{d s}{\lambda_{*}(s)}<\infty
$$

Отметим, что при выполнении условий (c)-(e)

$$
\mathbf{E} \int_{0}^{\tau}\left|\ln \lambda_{*}(t)\right| d N_{t}\left(\lambda_{0}\right)=\int_{0}^{\tau}\left|\ln \lambda_{*}(t)\right| \lambda_{0}(t) d t<\infty
$$

а поэтому для любой функции $\lambda_{1} \in K$

$$
\int_{0}^{\tau}\left|\ln \lambda_{1}(t)\right| d N_{t}\left(\lambda_{0}\right)<\infty \quad \text { п.н., }
$$

и отношение правдоподобия (2) корректно определено.

Обозначим $\alpha=\alpha(s, x):=a(s, x) / b(s), s \in \mathbf{R}^{+}, x \in \mathbf{R}^{+}$.

(f) Для любой функции $\lambda \in K \alpha(\cdot, \lambda(\cdot)) \in L_{\text {loc }}^{2}$ 
Построим ОМП функции $\lambda_{0}$ по наблюдениям (1) на отрезке $[0, T]$, $T \geqslant \tau$. Из равенства (2) следует, что для этого достаточно рассмотреть функционал, содержащий интегралы от наблюдаемых компонент $\left\{N_{t}\left(\lambda_{0}\right), \eta_{t}\left(\lambda_{0}\right)\right\}:$

$$
\begin{aligned}
Q_{T}(\lambda)= & Q_{T}(\lambda, \omega):=\frac{1}{T} \int_{0}^{T} \alpha(s, \lambda) d w_{s} \\
& -\frac{1}{2 T} \int_{0}^{T}\left[\alpha^{2}(s, \lambda)-2 \alpha\left(s, \lambda_{0}\right) \alpha(s, \lambda)\right] d s+\tilde{Q}_{T}(\lambda) .
\end{aligned}
$$

Здесь

$$
\widetilde{Q}_{T}(\lambda):=-\frac{1}{T} \int_{0}^{T} \lambda(s) d s+\frac{1}{T} \int_{0}^{T} \ln \lambda(s) d N_{s}\left(\lambda_{0}\right), \quad \lambda \in K .
$$

При выполнении условий (c)-(f) существует множество $\Omega_{0} \subset \Omega$ такое, что $\mathbf{P}\left(\Omega_{0}\right)=1$ и $Q_{T}(\lambda, \omega)$ принимает действительные значения для любого $T \geqslant \tau, \omega \in \Omega_{0}, \lambda \in K$. Непрерывность функционала $Q_{T}$ по $\lambda$ при $\omega \in \Omega_{0}$ обеспечивает следующее предположение.

(g) Функция $\alpha \in C^{1}\left(\mathbf{R}^{+} \times\left[\lambda_{*}, \lambda^{*}\right]\right)$, причем для любого $T>0$ сушествует $C_{T}>0$ такое, что при $s \leqslant T$

$$
\begin{aligned}
& \left|\frac{\partial \alpha(s, x)}{\partial s}-\frac{\partial \alpha(s, y)}{\partial s}\right| \leqslant C_{T}|x-y| \\
& \left|\frac{\partial \alpha(s, x)}{\partial x}-\frac{\partial \alpha(s, y)}{\partial x}\right| \leqslant C_{T}|x-y| .
\end{aligned}
$$

Далее любую постоянную, зависяшую лишь от $T$, обозначим через $C_{T}$ либо $D_{T}$; абсолютную постоянную обозначим через $C$ либо $C_{1}, C_{2}$. Одна и та же буква может быть использована для обозначения разных констант.

Лемма 1. При выполнении условий (c)-(e), (g) функционал $Q_{T}$ непрерывен по $\lambda \in K$ при $\omega \in \Omega_{0}$.

Д ок а $з$ а т е л ь с т в о. Непрерывность функционала $\widetilde{Q}_{T}(\lambda)$ очевидна. Далее, из условия (g) следует ограниченность функщий $\alpha$ и $\partial \alpha / \partial s$ на компакте $\widetilde{K}:=[0, T] \times\left[\lambda_{*}, \lambda^{*}\right]$. Поэтому при $\lambda_{1}, \lambda_{2} \in K$

$$
\begin{aligned}
& \left|\int_{0}^{T}\left[\alpha^{2}\left(s, \lambda_{1}\right)-\alpha^{2}\left(s, \lambda_{2}\right)\right] d s-2 \int_{0}^{T} \alpha\left(s, \lambda_{0}\right)\left[\alpha\left(s, \lambda_{1}\right)-\alpha\left(s, \lambda_{2}\right)\right] d s\right| \\
& \quad \leqslant C_{T} \int_{0}^{T}\left|\lambda_{1}(s)-\lambda_{2}(s)\right| d s \leqslant C_{T}\left(\left[\frac{T}{\tau}\right]+1\right)\left\|\lambda_{1}-\lambda_{2}\right\|_{\bar{W}_{2}^{1}} .
\end{aligned}
$$

Здесь и далее $[T / \tau]$ есть целая часть отношения $T / \tau$. Приращение стохастического интеграла в (3) представимо в виде:

$$
\int_{0}^{T}\left[\alpha\left(s, \lambda_{2}\right)-\alpha\left(s, \lambda_{1}\right)\right] d w_{s}=w_{T}\left[\alpha\left(T, \lambda_{2}(T)\right)-\alpha\left(T, \lambda_{1}(T)\right)\right]
$$




$$
\begin{aligned}
-\int_{0}^{T} w_{s} & {\left[\frac{\partial \alpha\left(s, \lambda_{2}\right)}{\partial s}-\frac{\partial \alpha\left(s, \lambda_{1}\right)}{\partial s}\right.} \\
& \left.+\frac{\partial \alpha\left(s, \lambda_{2}\right)}{\partial x} \lambda_{2}^{\prime}(s)-\frac{\partial \alpha\left(s, \lambda_{1}\right)}{\partial x} \lambda_{1}^{\prime}(s)\right] d s
\end{aligned}
$$

Отсюда с использованием замечания 1, a) имеем:

$$
\begin{aligned}
& \left|\int_{0}^{T}\left[\alpha\left(s, \lambda_{2}\right)-\alpha\left(s, \lambda_{1}\right)\right] d w_{s}\right| \leqslant\left|w_{T}\right| C_{T}\left|\lambda_{2}(T)-\lambda_{1}(T)\right| \\
& \quad+C_{T} \int_{0}^{T}\left|w_{s}\right|\left|\lambda_{2}(s)-\lambda_{1}(s)\right| d s \\
& \quad+\int_{0}^{T}\left|w_{s}\right| \frac{\partial \alpha\left(s, \lambda_{2}\right)}{\partial x}-\frac{\partial \alpha\left(s, \lambda_{1}\right)}{\partial x}|| \lambda_{2}^{\prime}(s) \mid d s \\
& \quad+C_{T} \int_{0}^{T}\left|w_{s}\right|\left|\lambda_{2}^{\prime}(s)-\lambda_{1}^{\prime}(s)\right| d s \leqslant \widetilde{C}_{T}\left(\left|w_{T}\right|+\bar{K} \sup _{s \leqslant T}\left|w_{s}\right|\right)\left\|\lambda_{2}-\lambda_{1}\right\|_{\bar{W}_{2}^{1}},
\end{aligned}
$$

где $\widetilde{C}_{T}$ - константа, отличная, вообце говоря, от $C_{T}$. Напомним, что величина $\bar{K}$ определялась сразу после условия (d). Из оценок (4)-(6) следует искомое утверждение.

Доказательство следующего результата состоит в применении теоремы об измеримом выборе [7] и аналогично доказательству существования оценки из работы [8].

Лемма 2. Пусть выполнены условия (c)-(e), (g). Тогда для каждого $T \geqslant \tau$ существует $K$-значный случайный элемент $\lambda_{T}=\lambda_{T}(\omega, s)$, $\omega \in \Omega, s \in[0, T]$, удовлетворяющий при каждом $\omega \in \Omega_{0}$ соотношению

$$
Q_{T}\left(\lambda_{T}\right)=\max _{\lambda \in K} Q_{T}(\lambda)
$$

О п р е д е л е н и е. Случайную функцию $\lambda_{T}$ из леммы 2 (продолженную с периодом $\tau$ на $\mathbf{R}^{+}$) назовем оценкой максимального правдоподобия интенсивности процесса $\left\{N_{t}\left(\lambda_{0}\right)\right\}$, построенной по наблюдениям $\left\{\xi_{t}, t \in[0, T]\right\}$.

3 а м е ч н и е 2. Вообще говоря, функция $\lambda_{T}$ определяется неединственным образом. Далее в качестве ОМП рассматривается одна из таких функций.

3 а м е ч а н и е 3. Алгоритм приближенного вычисления непараметрической ОМП, построенной по наблюдениям над пуассоновской компонентой, предложен в статье [3]. Аналогичная процедура применима к введенным оценкам $\lambda_{T}$ в комбинированной модели наблюдения.

3 а м е ч а н и е 4 . В силу упреждаемости $\lambda_{T}(s)$ слагаемое

$$
\int_{0}^{T} \alpha\left(s, \lambda_{T}(s)\right) d w_{s},
$$

входящее в значение $Q_{T}\left(\lambda_{T}\right)$, не является стохастическим интегралом Ито. Однако при выполнении ограничения (g) на гладкость функции $\alpha$ 
и при надлежащей гладкости $\lambda_{T}(s)$ этот интеграл можно трактовать с помощью формулы интегрирования по частям аналогично (5).

3. Состоятельность оценки. Предположим, что функция $\alpha$ удовлетворяет следующим условиям.

(h) $\alpha(s, x)=\sum_{i=1}^{3} \alpha_{i}(s, x), \quad$ где $\alpha_{i} \in C\left(\mathbf{R}^{+} \times\left[\lambda_{*}, \lambda^{*}\right]\right)$, причем:

1) при каждом $x \in\left[\lambda_{*}, \lambda^{*}\right] \alpha_{1}$ - периодическая по $s$ функция с периодом $\tau$;

2) $\max _{\lambda_{*}(s) \leqslant x \leqslant \lambda^{*}(s)}\left|\alpha_{2}(s, x)\right| \longrightarrow 0, \quad s \rightarrow \infty$

3) $\int_{1}^{\infty} \frac{\left(\alpha_{3}^{*}(s)\right)^{2}}{s} d s<\infty, \quad$ где $\alpha_{3}^{*}(s):=\max _{\lambda_{*}(s) \leqslant x \leqslant \lambda^{*}(s)}\left|\alpha_{3}(s, x)\right|$.

Таким образом, функция $\alpha$ разложена на 3 компоненты: периодическую по $s$, равномерно стремяшуюся к нулю при $s \rightarrow \infty$ и малую «в среднем» при $s \rightarrow \infty$. Введем предельный функционал

$$
Q_{\infty}(\lambda):=-\frac{1}{2 \tau} \int_{0}^{\tau}\left|\alpha_{1}(s, \lambda)\right|^{2} d s+\frac{1}{\tau} \int_{0}^{\tau} \alpha_{1}(s, \lambda) \alpha_{1}\left(s, \lambda_{0}\right) d s+\widetilde{Q}_{\infty}(\lambda)
$$

где

$$
\widetilde{Q}_{\infty}(\lambda):=-\frac{1}{\tau} \int_{0}^{\tau} \lambda(s) d s+\frac{1}{\tau} \int_{0}^{\tau} \ln \lambda(s) \lambda_{0}(s) d s ; \quad \lambda \in K .
$$

Теорема 1. Пусть выполиены условия (c)-(e), (g), (h). Тогда при каждом $\lambda \in K$

$$
Q_{T}(\lambda) \rightarrow Q_{\infty}(\lambda) \quad \text { (n.н.), } \quad T \rightarrow \infty
$$

Д о к а з а т е л ь с т в о. В статье [3] доказано, что при выполнении условий $\left(\right.$ c)-(e) $\widetilde{Q}_{T}(\lambda) \rightarrow \widetilde{Q}_{\infty}(\lambda)$ п.н. при $T \rightarrow \infty$. Рассмотрим сходимость остальных слагаемых функпионала (3). Положим

$$
Q_{T}^{i}(\lambda):=\frac{1}{T} \int_{0}^{T} \alpha_{i}(s, \lambda) d w_{s}, \quad \lambda \in K, 1 \leqslant i \leqslant 3 .
$$

При $T=n \tau, i=1$

$$
Q_{n \tau}^{1}(\lambda)=\frac{1}{n \tau} \sum_{i=1}^{n} \int_{(i-1) \tau}^{i \tau} \alpha_{1}(s, \lambda) d w_{s} \rightarrow 0 \quad \text { (п.н.), } \quad n \rightarrow \infty,
$$

в силу усиленного закона больших чисел (УЗБЧ) [9, с. 332, теорема 12] и неравенства $\mathbf{E}\left|\int_{0}^{\tau} \alpha_{1}(s, \lambda) d w_{s}\right| \leqslant\left(\int_{0}^{\tau} \alpha_{1}^{2}(s, \lambda) d s\right)^{1 / 2}<\infty$. 
Для оценки «остатка» $\left|Q_{T}^{1}(\lambda)-Q_{n \tau}^{1}(\lambda)\right|$ достаточно оценить выражение $n^{-1} \sup _{0 \leqslant h \leqslant \tau}\left|\int_{n \tau}^{n \tau+h} \alpha_{1}(s, \lambda) d w_{s}\right|$. В силу неравенства Дуба для мартингалов $[10$, c. 67$]$ и периодичности $\alpha_{1}$ имеем:

$$
\sum_{n=1}^{\infty} \frac{1}{n^{2}} \mathbf{E} \sup _{0 \leqslant h \leqslant \tau}\left|\int_{n \tau}^{n \tau+h} \alpha_{1}(s, \lambda) d w_{s}\right|^{2} \leqslant 4 \sum_{n=1}^{\infty} \frac{1}{n^{2}} \int_{0}^{\tau} \alpha_{1}^{2}(s, \lambda) d s<\infty .
$$

Поэтому

$$
\sum_{n=1}^{\infty} \frac{1}{n^{2}} \sup _{0 \leqslant h \leqslant \tau}\left|\int_{n \tau}^{n \tau+h} \alpha_{1}(s, \lambda) d w_{s}\right|^{2}<\infty \quad \text { (п.н.) }
$$

откуда

$$
\frac{1}{n} \sup _{0 \leqslant h \leqslant \tau}\left|\int_{n \tau}^{n \tau+h} \alpha_{1}(s, \lambda) d w_{s}\right| \longrightarrow 0 \quad \text { (п.н.), } n \rightarrow \infty .
$$

Итак, $Q_{T}^{1}(\lambda) \rightarrow 0$ (п.н.), $T \rightarrow \infty$.

Далее, по условию $(\mathrm{h}), 2)$

$$
\sum_{n=1}^{\infty} \frac{1}{n^{2}} \mathbf{E}\left|\int_{(n-1) \tau}^{n \tau} \alpha_{2}(s, \lambda) d w_{s}\right|^{2}=\sum_{n=1}^{\infty} \frac{1}{n^{2}} \int_{(n-1) \tau}^{n \tau}\left|\alpha_{2}(s, \lambda)\right|^{2} d s<\infty .
$$

Вновь применяя УЗБЧ $[9$, с. 332 , теорема 12$]$, получаем, что $Q_{n \tau}^{2}(\lambda) \rightarrow 0$ п.н., $n \rightarrow \infty$. «Остаток» $\left|Q_{T}^{2}(\lambda)-Q_{n \tau}^{2}(\lambda)\right|$ оценивается аналогично $(7)$; тогда $Q_{T}^{2}(\lambda) \rightarrow 0$ п.н. при $T \rightarrow \infty$.

Далее, в силу условия $(\mathrm{h}), 3)$ имеем:

$$
\sum_{n=1}^{\infty} \frac{1}{n^{2}} \int_{(n-1) \tau}^{n \tau} \alpha_{3}^{2}(s, \lambda) d s \leqslant \tau^{2} \int_{\tau}^{\infty} \frac{\alpha_{3}^{2}(s, \lambda)}{s^{2}} d s+\int_{0}^{\tau} \alpha_{3}^{2}(s, \lambda) d s<\infty,
$$

и согласно УЗБЧ $\left[9\right.$, с. 332 , теорема 12], $Q_{n \tau}^{3}(\lambda) \rightarrow 0$ п.н., $n \rightarrow \infty$. «Остаток» $\left|Q_{T}^{3}(\lambda)-Q_{n \tau}^{3}(\lambda)\right|$ оценивается аналогично $(7)$, и $Q_{T}^{3}(\lambda) \rightarrow 0$ п.н., $T \rightarrow \infty$.

Наконец, рассмотрим

$$
R_{T}(\lambda):=\frac{1}{T} \int_{0}^{T}\left[\alpha^{2}(s, \lambda)-2 \alpha(s, \lambda) \alpha\left(s, \lambda_{0}\right)\right] d s .
$$

При выполнении условия (h) имеем при $T \rightarrow \infty$ :

$$
\begin{aligned}
& \frac{1}{T} \int_{0}^{T} \alpha_{1}^{2}(s, \lambda) d s \longrightarrow \frac{1}{\tau} \int_{0}^{\tau} \alpha_{1}^{2}(s, \lambda) d s ; \quad \frac{1}{T} \int_{0}^{T} \alpha_{2}^{2}(s, \lambda) d s \longrightarrow 0 ; \\
& \lim _{T \rightarrow \infty} \frac{1}{T} \int_{1}^{T} \alpha_{3}^{2}(s, \lambda) d s \\
& \quad=\lim _{T \rightarrow \infty}\left[\int_{1}^{T} \frac{\alpha_{3}^{2}(s, \lambda)}{s} d s-\frac{1}{T} \int_{1}^{T}\left(\int_{1}^{z} \frac{\alpha_{3}^{2}(s, \lambda)}{s} d s\right) d z\right] \\
& \quad=\int_{0}^{\infty} \frac{\alpha_{3}^{2}(s, \lambda)}{s} d s-\lim _{z \rightarrow \infty} \int_{1}^{z} \frac{\alpha_{3}^{2}(s, \lambda)}{s} d s=0 .
\end{aligned}
$$


Остальные слагаемые в (8) оцениватотся аналогично, тогда при $T \rightarrow \infty$

$$
R_{T}(\lambda) \longrightarrow \frac{1}{\tau} \int_{0}^{\tau}\left|\alpha_{1}(s, \lambda)\right|^{2} d s-\frac{2}{\tau} \int_{0}^{\tau} \alpha_{1}(s, \lambda) \alpha_{1}\left(s, \lambda_{0}\right) d s .
$$

Сходимость $Q_{T}(\lambda)$ к $Q_{\infty}(\lambda)$ следует теперь из полученных предельных соотношений.

Теорема 2. Пусть выполнены условия (c)-(e), (g), (h), а также следующее условие (i):

1) $\alpha_{1} \in C^{1}\left([0, \tau] \times\left[\lambda_{*}, \lambda^{*}\right]\right)$

2) $\alpha_{i} \in C^{1}\left(\mathbf{R}^{+} \times\left[\lambda_{*}, \lambda^{*}\right]\right)$ и для некоторой ограниченной функчии $C_{s}$

$$
\begin{gathered}
\left|\frac{\partial \alpha_{i}(s, x)}{\partial x}\right| \leqslant C_{s}, \\
\left|\frac{\partial \alpha_{i}(s, x)}{\partial p}-\frac{\partial \alpha_{i}(s, y)}{\partial p}\right| \leqslant C_{s}|x-y|,
\end{gathered}
$$

где $p=s, x ; i=2,3, s \in \mathbf{R}^{+} ; x, y \in\left[\lambda_{*}, \lambda^{*}\right]$, причем

3) $\varlimsup_{T \rightarrow \infty} \frac{1}{T} \int_{e}^{T} s(\ln \ln s) C_{s}^{2} d s<\infty \quad u$

4) $\lim _{t \rightarrow \infty} \sqrt{\frac{\ln \ln t}{t}} \alpha_{3}^{*}(t)=0$.

Тогда оченка $\lambda_{T}$ состоятельна в следуюием смысле:

$$
\left\|\lambda_{T}-\lambda_{0}\right\|_{\bar{W}_{2}^{1}} \longrightarrow 0 \quad \text { n.н., } T \rightarrow \infty .
$$

Д о к аз а т е л ь с т в о. Согласно общим условиям состоятельности $[1$, с. 76$]$, для выполнения (9) достаточно, чтобы выполнялись условия: a) $Q_{T}(\lambda) \rightarrow Q_{\infty}(\lambda), T \rightarrow \infty$ п.н.

$$
\text { b) } \quad Q_{\infty}\left(\lambda_{0}\right)>Q_{\infty}(\lambda), \quad \lambda \in K, \lambda \neq \lambda_{0}
$$

c) сушествует такая функщия $\omega(\gamma), \gamma>0$, что $\omega(\gamma) \rightarrow 0, \gamma \rightarrow 0$ и

$$
\mathbf{P}\left\{\varlimsup_{T \rightarrow \infty} \sup _{\lambda_{1}, \lambda_{2} \in K,\left\|\lambda_{1}-\lambda_{2}\right\|_{\bar{W}_{2}^{1}} \leqslant \gamma}\left|Q_{T}\left(\lambda_{2}\right)-Q_{T}\left(\lambda_{1}\right)\right| \leqslant \omega(\gamma)\right\}=1 .
$$

Сходимость а) имеет место по теореме 1 ; неравенство (10) следует из представления

$$
Q_{\infty}(\lambda)=-\frac{1}{2 \tau} \int_{0}^{\tau}\left|\alpha_{1}(s, \lambda)-\alpha_{1}\left(s, \lambda_{0}\right)\right|^{2} d s+\widetilde{Q}_{\infty}(\lambda)+\frac{1}{2 \tau} \int_{0}^{\tau} \alpha_{1}^{2}\left(s, \lambda_{0}\right) d s
$$

и строгого неравенства $\widetilde{Q}_{\infty}(\lambda)<\widetilde{Q}_{\infty}\left(\lambda_{0}\right), \lambda \neq \lambda_{0}$. 
Соотношение (11) доказано в [3] для функционала $\widetilde{Q}_{T}(\lambda)$. Поэтому достаточно установить (11) для функционала

$$
\check{Q}_{T}(\lambda):=T^{-1} \int_{0}^{T}\left|\alpha(s, \lambda)-\alpha\left(s, \lambda_{0}\right)\right|^{2} d s
$$

и функционалов $Q_{T}^{i}$, введенных при доказательстве теоремы 1.

1) Оценим $Q_{T}^{1}\left(\lambda_{1}\right)-Q_{T}^{1}\left(\lambda_{2}\right)$ при $T=n \tau$ :

$$
\begin{gathered}
\left|Q_{n \tau}^{1}\left(\lambda_{1}\right)-Q_{n \tau}^{1}\left(\lambda_{2}\right)\right| \leqslant \frac{1}{n \tau}\left|B_{n}(\tau)\right|\left|\alpha_{1}\left(\tau, \lambda_{1}(\tau)\right)-\alpha_{1}\left(\tau, \lambda_{2}(\tau)\right)\right| \\
\quad+\frac{1}{n \tau} \int_{0}^{\tau}\left|B_{n}(s)\right|\left|\frac{d}{d s}\left(\alpha_{1}\left(s, \lambda_{1}(s)\right)-\alpha_{1}\left(s, \lambda_{2}(s)\right)\right)\right| d s
\end{gathered}
$$

где

$$
B_{n}(s):=\sum_{i=0}^{n-1}(w(s+i \tau)-w(i \tau)), \quad s \in[0, \tau] .
$$

По УЗБЧ [9, с. 332, теорема 12] $n^{-1}\left|B_{n}(\tau)\right| \rightarrow 0$ п.н., $n \rightarrow \infty$, и первое слагаемое правой части (12) равномерно по $\lambda_{1}, \lambda_{2} \in K$ стремится к нулю п.н. Далее,

$$
\begin{gathered}
\frac{1}{n \tau} \int_{0}^{\tau}\left|B_{n}(s)\right|\left|\frac{d}{d s}\left(\alpha_{1}\left(s, \lambda_{1}(s)\right)-\alpha_{1}\left(s, \lambda_{2}(s)\right)\right)\right| d s \leqslant \frac{1}{n \tau}\left(\int_{0}^{\tau} B_{n}^{2}(s) d s\right)^{1 / 2} \\
\quad \times\left(\int_{0}^{\tau}\left|\frac{d}{d s}\left(\alpha_{1}\left(s, \lambda_{1}(s)\right)-\alpha_{1}\left(s, \lambda_{2}(s)\right)\right)\right|^{2} d s\right)^{1 / 2}
\end{gathered}
$$

Обозначим $Y_{i}(s):=w(s+i \tau)-w(i \tau), s \in[0, \tau],-$ независимые центрированные одинаково распределенные гауссовские элементы в пространстве $L_{2}[0, \tau]$. В силу УЗБЧ в гильбертовом пространстве, доказанного в работе $[11$, с. 145$]$, имеем:

$$
\frac{1}{n}\left(\int_{0}^{\tau} B_{n}^{2}(s) d s\right)^{1 / 2}=\left\|\frac{1}{n} \sum_{i=0}^{n-1} Y_{i}\right\|_{L_{2}[0, \tau]} \longrightarrow 0 \quad \text { п.н., } n \rightarrow \infty .
$$

Кроме того, в силу условия (i), 1)

$$
\begin{aligned}
\int_{0}^{\tau}\left|\frac{d}{d s} \alpha_{1}\left(s, \lambda_{1}(s)\right)\right|^{2} d s & \leqslant 2 \int_{0}^{\tau}\left|\frac{\partial \alpha_{1}\left(s, \lambda_{1}\right)}{\partial s}\right|^{2} d s+2 \int_{0}^{\tau}\left|\frac{\partial \alpha_{1}\left(s, \lambda_{1}\right)}{\partial x}\right|^{2}\left|\lambda_{1}^{\prime}(s)\right|^{2} d s \\
& \leqslant C_{1}+C_{2}\left\|\lambda_{1}\right\|_{\bar{W}_{2}^{1}}^{2} \leqslant C .
\end{aligned}
$$

Аналогичная оценка справедлива и для интеграла

$$
\int_{0}^{\tau}\left|\frac{d}{d s} \alpha_{1}\left(s, \lambda_{2}(s)\right)\right|^{2} d s
$$

Итак, из оценок (12)-(15) следует, что

$$
\varlimsup_{n \rightarrow \infty} \sup _{\lambda_{1}, \lambda_{2} \in K}\left|Q_{n \tau}^{1}\left(\lambda_{1}\right)-Q_{n \tau}^{1}\left(\lambda_{2}\right)\right|=0 \quad \text { п.н. }
$$


С учетом (15) «остаток» оценим так:

$$
\begin{aligned}
& \sup _{\lambda \in K} \sup _{0 \leqslant h \leqslant \tau} \frac{1}{n}\left|\int_{n \tau}^{n \tau+h} \alpha_{1}(s, \lambda) d w_{s}\right| \\
& \quad \leqslant C \sup _{0 \leqslant h \leqslant \tau} \frac{1}{n}(|w(n \tau+h)|+|w(n \tau)|)+C \frac{1}{n}\left(\int_{n \tau}^{(n+1) \tau} w_{s}^{2} d s\right)^{1 / 2} .
\end{aligned}
$$

Согласно закону повторного логарифма,

$$
\mathbf{P}\left\{\varlimsup_{t \rightarrow \infty} \frac{|w(t)|}{\sqrt{t \ln \ln t}}<\infty\right\}=1 .
$$

Оценивая $|w(t)|$ сверху через $\sqrt{t \ln \ln t}$, нетрудно показать, что правая часть (16) стремится к нулю п.н. при $n \rightarrow \infty$. Значит, доказано соотношение $\varlimsup_{T \rightarrow \infty} \sup _{\lambda_{1}, \lambda_{2} \in K}\left|Q_{T}^{1}\left(\lambda_{1}\right)-Q_{T}^{1}\left(\lambda_{2}\right)\right|=0$ п.н.

2) Оценим $\left|Q_{T}^{2}\left(\lambda_{1}\right)-Q_{T}^{2}\left(\lambda_{2}\right)\right|$ :

$$
\begin{aligned}
\left|Q_{T}^{2}\left(\lambda_{1}\right)-Q_{T}^{2}\left(\lambda_{2}\right)\right| \leqslant & \frac{1}{T}\left|w_{T}\right|\left|\alpha_{2}\left(T, \lambda_{1}(T)\right)-\alpha_{2}\left(T, \lambda_{2}(T)\right)\right| \\
& +\frac{1}{T} \int_{0}^{T}\left|w_{s}\right|\left|\frac{d}{d s}\left(\alpha_{2}\left(s, \lambda_{1}\right)-\alpha_{2}\left(s, \lambda_{2}\right)\right)\right| d s .
\end{aligned}
$$

По условию (h), 2) первое слагаемое правой части (17) равномерно по $\lambda_{1}, \lambda_{2} \in K$ стремится к нулю п.н. при $T \rightarrow \infty$. Второе слагаемое не превосходит суммы

$$
\begin{aligned}
& \frac{1}{T} \int_{0}^{T}\left|w_{s}\right|\left|\frac{\partial}{\partial s} \alpha_{2}\left(s, \lambda_{1}\right)-\frac{\partial}{\partial s} \alpha_{2}\left(s, \lambda_{2}\right)\right| d s \\
& \quad+\frac{1}{T} \int_{0}^{T}\left|w_{s}\right|\left|\frac{\partial}{\partial x} \alpha_{2}\left(s, \lambda_{1}\right)-\frac{\partial}{\partial x} \alpha_{2}\left(s, \lambda_{2}\right)\right|\left|\lambda_{1}^{\prime}(s)\right| d s \\
& \quad+\frac{1}{T} \int_{0}^{T}\left|w_{s}\right|\left|\frac{\partial}{\partial x} \alpha_{2}\left(s, \lambda_{2}\right)\right|\left|\lambda_{2}^{\prime}(s)-\lambda_{1}^{\prime}(s)\right| d s
\end{aligned}
$$

В силу условия (i), 2) при $T \in[n \tau,(n+1) \tau]$ имеем:

$$
\begin{aligned}
& \frac{1}{T} \int_{0}^{T}\left|w_{s}\right|\left|\frac{\partial}{\partial s} \alpha_{2}\left(s, \lambda_{1}\right)-\frac{\partial}{\partial s} \alpha_{2}\left(s, \lambda_{2}\right)\right| d s \\
& \quad \leqslant \frac{C}{\sqrt{T}}\left(\int_{0}^{T} w_{s}^{2} C_{s}^{2} d s\right)^{1 / 2}\left\|\lambda_{2}-\lambda_{1}\right\|_{\bar{W}_{2}^{1}}
\end{aligned}
$$

Отметим, что при каждом $A>0 \quad T^{-1} \int_{0}^{A} w_{s}^{2} d s \longrightarrow 0$ п.н., $T \rightarrow \infty$, и в силу условий (i), 2), 3) и закона повторного логарифма с вероятностью 1

$$
\varlimsup_{T \rightarrow \infty} \frac{1}{T} \int_{0}^{T} w_{s}^{2} C_{s}^{2} d s \leqslant \varlimsup_{T \rightarrow \infty} \frac{2}{T} \int_{e}^{T} s(\ln \ln s) C_{s}^{2} d s<\infty .
$$


Далее, согласно замечанию 1, а),

$$
\begin{aligned}
& \frac{1}{T} \int_{0}^{T}\left|w_{s}\right|\left|\frac{\partial \alpha_{2}\left(s, \lambda_{1}\right)}{\partial x}-\frac{\partial \alpha_{2}\left(s, \lambda_{2}\right)}{\partial x}\right|\left|\lambda_{1}^{\prime}(s)\right| d s \\
& \quad \leqslant \frac{1}{T} \int_{0}^{T}\left|w_{s}\right| C_{s}\left|\lambda_{1}(s)-\lambda_{2}(s)\right|\left|\lambda_{1}^{\prime}(s)\right| d s \\
& \quad \leqslant C \bar{K}\left(\frac{1}{T} \int_{0}^{T} w_{s}^{2} C_{s}^{2} d s\right)^{1 / 2}\left\|\lambda_{2}-\lambda_{1}\right\|_{\bar{W}_{2}^{1}},
\end{aligned}
$$

что дает нужные оценки в силу (19). Здесь $\bar{K}-$ константа, введенная B II. 2 .

Наконец,

$$
\begin{aligned}
& \frac{1}{T} \int_{0}^{T}\left|w_{s}\right|\left|\frac{\partial \alpha_{2}\left(s, \lambda_{2}\right)}{\partial x}\right|\left|\lambda_{2}^{\prime}(s)-\lambda_{1}^{\prime}(s)\right| d s \\
& \quad \leqslant C\left(\frac{1}{T} \int_{0}^{T} w_{s}^{2}\left|\frac{\partial \alpha_{2}\left(s, \lambda_{2}\right)}{\partial x}\right|^{2} d s\right)^{1 / 2}\left\|\lambda_{2}-\lambda_{1}\right\|_{\bar{W}_{2}^{1}} \\
& \quad \leqslant C\left(\frac{1}{T} \int_{0}^{T} w_{s}^{2} C_{s}^{2} d s\right)^{1 / 2}\left\|\lambda_{2}-\lambda_{1}\right\|_{\bar{W}_{2}^{1}} .
\end{aligned}
$$

Из полученных оценок вытекает соотношение (11) для $Q_{T}^{2}(\lambda)$ с линейной функцией $\omega(\gamma)$.

Аналогично, с использованием условия i), 2), 4) оцениваются приращения компоненты $Q_{T}^{3}(\lambda)$.

3) Оценим

$$
\begin{aligned}
& \left|\check{Q}_{T}\left(\lambda_{1}\right)-\check{Q}_{T}\left(\lambda_{2}\right)\right| \\
& \quad \leqslant 4\left(\frac{1}{T} \int_{0}^{T}\left|\alpha\left(s, \lambda_{1}\right)-\alpha\left(s, \lambda_{2}\right)\right|^{2} d s\right)^{1 / 2} \sup _{\lambda \in K}\left(\frac{1}{T} \int_{0}^{T}|\alpha(s, \lambda)|^{2} d s\right)^{1 / 2} .
\end{aligned}
$$

При выполнении условий теоремы для некоторого $C>0$

$$
\begin{array}{r}
\frac{1}{T} \int_{0}^{T}\left|\alpha_{i}(s, \lambda)\right|^{2} d s \leqslant C, \quad 1 \leqslant i \leqslant 3, \\
\frac{1}{T} \int_{0}^{T}\left|\alpha\left(s, \lambda_{1}\right)-\alpha\left(s, \lambda_{2}\right)\right|^{2} d s \leqslant C\left\|\lambda_{2}-\lambda_{1}\right\|_{\bar{W}_{2}^{1}}^{2},
\end{array}
$$

откуда получаем (11) для $\check{Q}_{T}(\lambda)$, и теорема доказана.

3 а м е ч а н и е 5 . Пусть $K-$ компакт в пространстве $\bar{W}_{2 p}^{1}$ при некотором $p>1$ и выполнены все условия теоремы 2. Тогда опенка $\lambda_{T}$ состоятельна в $\bar{W}_{2 p}^{1}$, т.е. $\left\|\lambda_{T}-\lambda_{0}\right\|_{\bar{W}_{2 p}^{1}} \longrightarrow 0$ I.н., $T \rightarrow \infty$. В самом деле, в силу замечания 1, a)

$$
\|f\|_{\bar{W}_{2}^{1}} \leqslant C_{p}\|f\|_{\bar{W}_{2 p}^{1}}, \quad f \in \bar{W}_{2}^{1},
$$


поэтому

$$
\begin{aligned}
& \mathbf{P}\left\{\varlimsup_{T \rightarrow \infty} \sup _{\lambda_{1}, \lambda_{2} \in K,\left\|\lambda_{1}-\lambda_{2}\right\|_{W_{2 p}}^{1} \leqslant \gamma}\left|Q_{T}\left(\lambda_{2}\right)-Q_{T}\left(\lambda_{1}\right)\right| \leqslant \omega(\gamma)\right\} \\
& \quad \geqslant \mathbf{P}\left\{\varlimsup_{T \rightarrow \infty} \sup _{\lambda_{1}, \lambda_{2} \in K,\left\|\lambda_{1}-\lambda_{2}\right\|_{\bar{W}_{2}^{1}}^{1} \leqslant C_{p} \gamma}\left|Q_{T}\left(\lambda_{2}\right)-Q_{T}\left(\lambda_{1}\right)\right| \leqslant \omega(\gamma)\right\}=1 .
\end{aligned}
$$

Кроме того, функционал $Q_{T}$ непрерывен по $\lambda \in \bar{W}_{2 p}^{1}$; состоятельность в $\bar{W}_{2 p}^{1}$ следует из $[1$, с. 76$]$.

4. Скорость среднеквадратичной сходимости оценки. Введем дополнительные ограничения на параметрическое множество $K$ и функцию интенснвности $\lambda_{0}$.

(j) Множество $K$ компактно в пространстве $\bar{W}_{2 p}^{1}[0, \tau]$ при некотором $p \in(1,2)$ и ограничено в пространстве $\bar{W}_{2 q}^{1}[0, \tau]$, где $p, q-$ сопряженные индексы, причем все функции из $K$ неотрицательны. $[0, \tau]$.

(k) Функция $\lambda_{0}$ принадлежит $K$ и положительна при каждом $t \in$

Далее случайный процесс $\left\{x_{T}, T \geqslant 0\right\}$ будем обозначать через $O_{p}(1)$, если $\lim _{C \rightarrow \infty} \sup _{T \geqslant T_{0}} \mathbf{P}\left\{\left|x_{T}\right| \geqslant C\right\}=0$ при некотором $T_{0}>0$, и - через $o_{p}(1)$, если $x_{T} \stackrel{\mathbf{P}}{\longrightarrow} 0, T \rightarrow \infty$. При написании введенных символов указание, что $T \rightarrow \infty$, будет опускаться.

Теорема 3. Пусть выполнены условия (c), (e), (g)-(i), (j), (k), a также следующее условие (1).

1) Для некоторой постоянной $C$

$$
\left|\frac{\partial \alpha_{1}(s, x)}{\partial p}-\frac{\partial \alpha_{1}(s, y)}{\partial p}\right| \leqslant C|x-y|
$$

гдe $p=s, x ; s \in[0, \tau], x, y \in\left[\lambda_{*}, \lambda^{*}\right]$

2) для величины $C_{s}$ из условия (i) $\int_{0}^{\infty} s C_{s}^{2} d s<\infty$, причем для этой величины выполнены также неравенства

$$
\left|\frac{\partial \alpha_{i}(s, x)}{\partial s}\right| \leqslant C_{s}, \quad i=2,3, s \in \mathbf{R}^{+}, x \in\left[\lambda_{*}, \lambda^{*}\right] .
$$

Tozda

$$
T^{1 / 2} \int_{0}^{T}\left|\lambda_{T}(t)-\lambda_{0}(t)\right|^{2} d t=o_{p}(1)
$$

Д ок а 3 ательст в о. Согласно теореме 2 и замечанию 5, при выполнении условия (j) $\left\|\lambda_{T}-\lambda_{0}\right\|_{\bar{W}_{2 p}^{1}} \longrightarrow 0$ п.н. при $T \rightarrow \infty$. Из теоремы вложения соболевских пространств (см. замечание 1,6$)$ ) и условия $(\mathrm{k})$ имеем:

$$
\max _{0 \leqslant t \leqslant \tau} \frac{\left|\lambda_{T}(t)-\lambda_{0}(t)\right|}{\lambda_{0}(t)} \rightarrow 0 \quad \text { (п.н.), } T \rightarrow \infty \text {. }
$$


Обозначим

$$
\begin{aligned}
\Delta_{T}(t) & :=\lambda_{T}(t)-\lambda_{0}(t) ; \quad a_{0}(t):=\int_{0}^{t} \lambda_{0}(u) d u \\
N_{i}(t) & :=N(t+\tau(i-1))-N(\tau(i-1)), \quad i \geqslant 1,
\end{aligned}
$$

где $N(t)=N_{t}\left(\lambda_{0}\right) ; A_{n}(t):=n^{-1} \sum_{i=1}^{n} N_{i}(t)-a_{0}(t) ; t \in[0, \tau]$. Перепишем функционал $Q_{T}$ в виде

$$
\begin{aligned}
Q_{T}(\lambda)=\frac{1}{T} & \int_{0}^{n \tau} \alpha(s, \lambda) d w_{s}-\frac{1}{2 T} \int_{0}^{T}\left|\alpha(s, \lambda)-\alpha\left(s, \lambda_{0}\right)\right|^{2} d s \\
& +\frac{1}{2 T} \int_{0}^{T} \alpha^{2}\left(s, \lambda_{0}\right) d s \\
& +\frac{n}{T}\left[-\int_{0}^{\tau} \lambda(s) d s+\int_{0}^{\tau} \lambda_{0}(t) \ln \lambda(t) d t+\int_{0}^{\tau} \ln \lambda(t) d A_{n}(t)\right] \\
& +R_{T}^{1}(\lambda)+R_{T}^{2}(\lambda),
\end{aligned}
$$

где

$$
\begin{aligned}
& R_{T}^{1}(\lambda):=-\frac{1}{T} \int_{0}^{T-n \tau} \lambda(t) d t+\frac{1}{T} \int_{0}^{T-n \tau} \ln \lambda(t) d N_{n+1}(t), \\
& R_{T}^{2}(\lambda):=\frac{1}{T} \int_{n \tau}^{T} \alpha(s, \lambda) d w_{s}, \quad n \tau \leqslant T<(n+1) \tau .
\end{aligned}
$$

По определению оценки $Q_{T}\left(\lambda_{T}\right) \geqslant Q_{T}\left(\lambda_{0}\right)$, откуда

$$
\begin{aligned}
\int_{0}^{\tau} & \Delta_{T}(t) d t-\int_{0}^{\tau} \lambda_{0}(t) \ln \left(1+\frac{\Delta_{T}(t)}{\lambda_{0}(t)}\right) d t \\
& \leqslant \int_{0}^{\tau} \ln \left(1+\frac{\Delta_{T}(t)}{\lambda_{0}(t)}\right) d A_{n}(t)+\frac{1}{n} \int_{0}^{n \tau}\left[\alpha\left(s, \lambda_{T}\right)-\alpha\left(s, \lambda_{0}\right)\right] d w_{s} \\
& -\frac{1}{2 n} \int_{0}^{n \tau}\left|\alpha\left(s, \lambda_{T}\right)-\alpha\left(s, \lambda_{0}\right)\right|^{2} d s+\frac{T}{n} \sum_{i=1,2}\left(\left|R_{T}^{i}\left(\lambda_{T}\right)\right|+\left|R_{T}^{i}\left(\lambda_{0}\right)\right|\right) .
\end{aligned}
$$

В статье [3] доказано, что при выполнении условий теоремы $R_{T}^{1}\left(\lambda_{T}\right)$ и $R_{T}^{1}\left(\lambda_{0}\right)$ есть $O_{p}(1) / T$. Кроме того, из $(20)$ следует существование такого $\delta>0$, что

$$
\lim _{T \rightarrow \infty} \mathbf{P}\left\{\int_{0}^{\tau}\left[\Delta_{T}(t)-\lambda_{0}(t) \ln \left(1+\frac{\Delta_{T}(t)}{\lambda_{0}(t)}\right)\right] d t \geqslant \delta \int_{0}^{\tau}\left|\Delta_{T}(t)\right|^{2} d t\right\}=1 .
$$

В работе [3] доказано также, что

$$
\int_{0}^{\tau} \ln \left(1+\frac{\Delta_{T}(t)}{\lambda_{0}(t)}\right) d A_{n}(t)=\frac{o_{p}(1)}{\sqrt{T}}
$$

Оџеним

$$
Q_{n \tau}^{i}\left(\lambda_{T}\right)-Q_{n \tau}^{i}\left(\lambda_{0}\right)=\frac{1}{n \tau} \int_{0}^{n \tau}\left[\alpha_{i}\left(s, \lambda_{T}\right)-\alpha_{i}\left(s, \lambda_{0}\right)\right] d w_{s},
$$


используя (12)-(15), (17), (18) и условие (1):

$$
\begin{aligned}
& \left|Q_{n \tau}^{1}\left(\lambda_{T}\right)-Q_{n \tau}^{1}\left(\lambda_{0}\right)\right| \leqslant \frac{1}{n \tau}\left|B_{n}(\tau)\right| C\left|\lambda_{T}(\tau)-\lambda_{0}(\tau)\right| \\
& \quad+\frac{1}{\sqrt{n} \tau}\left\|\frac{1}{\sqrt{n}} \sum_{i=0}^{n-1} Y_{i}\right\| \|_{L_{2}[0, \tau]}\left(\int _ { 0 } ^ { \tau } \left[\left|\frac{\partial}{\partial s} \alpha_{1}\left(s, \lambda_{T}\right)-\frac{\partial}{\partial s} \alpha_{1}\left(s, \lambda_{0}\right)\right|\right.\right. \\
& \quad+\left|\frac{\partial}{\partial x} \alpha_{1}\left(s, \lambda_{T}\right)-\frac{\partial}{\partial x} \alpha_{1}\left(s, \lambda_{0}\right)\right|\left|\lambda_{0}^{\prime}(s)\right| \\
& \left.\left.\quad+\left|\frac{\partial}{\partial x} \alpha_{1}\left(s, \lambda_{T}\right)\right|\left|\lambda_{T}^{\prime}(s)-\lambda_{0}^{\prime}(s)\right|\right]^{2} d s\right)^{1 / 2}
\end{aligned}
$$

Далее,

$$
\frac{1}{\sqrt{n}}\left|B_{n}(\tau)\right|=O_{p}(1), \quad\left\|\frac{1}{\sqrt{n}} \sum_{i=0}^{n-1} Y_{i}\right\|_{L_{2}[0, \tau]}=O_{p}(1) .
$$

По условию (1), 1)

$$
\left(\int_{0}^{\tau}\left|\frac{\partial}{\partial s}\left(\alpha_{1}\left(s, \lambda_{T}\right)-\alpha_{1}\left(s, \lambda_{0}\right)\right)\right|^{2} d s\right)^{1 / 2}=o_{p}(1) .
$$

По условиям (j) и (1), 1)

$$
\begin{aligned}
& \left(\int_{0}^{\tau}\left|\frac{\partial}{\partial x}\left(\alpha_{1}\left(s, \lambda_{T}\right)-\alpha_{1}\left(s, \lambda_{0}\right)\right)\right|^{2}\left|\lambda_{0}^{\prime}(s)\right|^{2} d s\right)^{1 / 2} \\
& \quad \leqslant C\left(\int_{0}^{\tau}\left|\lambda_{T}-\lambda_{0}\right|^{2 p} d s\right)^{1 /(2 p)}\left(\int_{0}^{\tau}\left|\lambda_{0}^{\prime}(s)\right|^{2 q} d s\right)^{1 /(2 q)} \\
& \quad \leqslant C\left\|\lambda_{T}-\lambda_{0}\right\|_{\bar{W}_{2 p}^{1}}=o_{p}(1) .
\end{aligned}
$$

Далее,

$$
\left(\int_{0}^{\tau}\left|\frac{\partial}{\partial x} \alpha_{1}\left(s, \lambda_{T}\right)\right|^{2}\left|\lambda_{T}^{\prime}(s)-\lambda_{0}^{\prime}(s)\right|^{2} d s\right)^{1 / 2} \leqslant C\left\|\lambda_{T}-\lambda_{0}\right\|_{\bar{W}_{2 p}^{1}}=o_{p}(1) .
$$

Согласно (24)-(27), левая часть (23) есть $o_{p}(1) / \sqrt{T}$. Из соотношений $(17),(18)$ и условия $(1), 2)$ имеем для $i=2,3$

$$
\begin{aligned}
& \left|Q_{n \tau}^{i}\left(\lambda_{T}\right)-Q_{n \tau}^{i}\left(\lambda_{0}\right)\right| \leqslant \frac{1}{\sqrt{n \tau}} \frac{\left|w_{n \tau}\right|}{\sqrt{n \tau}}\left|\alpha_{i}\left(n \tau, \lambda_{T}(n \tau)\right)-\alpha_{i}\left(n \tau, \lambda_{0}(n \tau)\right)\right| \\
& \quad+\frac{C}{\sqrt{n}}\left(\int_{0}^{n \tau} w_{s}^{2} C_{s}^{2} d s\right)^{1 / 2}\left\|\lambda_{T}-\lambda_{0}\right\|_{\bar{W}_{2}^{1}} \\
& \quad+\frac{C}{n}\left(\int_{0}^{n \tau} w_{s}^{2} C_{s}^{2} d s\right)^{1 / 2}\left\|\lambda_{T}-\lambda_{0}\right\|_{\bar{W}_{2}^{1}}=\frac{o_{p}(1)}{\sqrt{T}}
\end{aligned}
$$


Мы воспользовались оценкой:

$$
\mathrm{E} \int_{0}^{n \tau} w_{s}^{2} C_{s}^{2} d s=\int_{0}^{n \tau} s C_{s}^{2} d s \leqslant \int_{0}^{\infty} s C_{s}^{2} d s<\infty, \quad n \geqslant 1
$$

Рассмотрим остаток $R_{T}^{2}\left(\lambda_{T}\right)$ (остаток $R_{T}^{2}\left(\lambda_{0}\right)$ оценивается аналогично):

$$
\begin{aligned}
\left|R_{T}^{2}\left(\lambda_{T}\right)\right| \leqslant & \frac{1}{T}|w(T)-w(n \tau)| \\
& \times\left(\sup _{0 \leqslant s \leqslant \tau, x \in\left[\lambda_{*}, \lambda^{*}\right]}\left|\alpha_{1}(s, x)\right|+\sup _{i=2,3, n \tau \leqslant s \leqslant T, x \in\left[\lambda_{*}, \lambda^{*}\right]}\left|\alpha_{i}(s, x)\right|\right) \\
& +\frac{1}{T} \int_{n \tau}^{T}\left|w_{s}\right|\left|\frac{d}{d s} \alpha\left(s, \lambda_{T}(s)\right)\right| d s .
\end{aligned}
$$

По условию (1)

$$
\left|R_{T}^{2}\left(\lambda_{T}\right)\right|=\frac{o_{p}(1)}{\sqrt{T}}
$$

Из оценок (21)-(29) следует теперь утверждение теоремы.

3 а м е ч а и и е 6 . Если функция $\alpha_{1}(s, x)$ линейна по $x$, то вместо условия (j) достаточно потребовать (d). В самом деле, тогда левая часть (26) равна нулю, а остальные слагаемые в правой части (23) оцениваются через

$$
\frac{O_{p}(1)}{\sqrt{T}}\left\|\lambda_{T}-\lambda_{0}\right\|_{W_{2}^{1}}
$$

5. Сходимость гладких функционалов от оценки. Пусть $p \in$ $(1,2), q$ - сопряженный индекс; $K_{0}$ - открытое ограниченное выпуклое множество в $\bar{W}_{2 q}^{1}$, состояшее из положительных функций; $K$ - замыкание $K_{0}$ в пространстве $\bar{W}_{2 p}^{1}$. Поскольку $p<q$, то $\bar{W}_{2 p}^{1}$ компактно вложено в $\bar{W}_{2 q}^{1}$ (замечание $\left.1, \sigma\right)$ ), и $K$ - компакт в $\bar{W}_{2 p}^{1}$. Кроме того, $K$ - ограниченное множество в $\bar{W}_{2 q}^{1}$. В самом деле, пусть $\sup _{f \in K_{0}} \int_{0}^{\tau}\left|f^{\prime}\right|^{2 q} d s \leqslant R$. Если $\left\{f_{n}\right\} \subset K_{0}$ и $f_{n} \rightarrow g$ в $\bar{W}_{2 p}^{1}$, то $f_{n}$ сходится к $g$ равномерно на $[0, \tau]$; тогда в силу леммы $[10$, с. 86$]$, верной при показателе $2 q>1$, из неравенства $\int_{0}^{\tau}\left|f_{n}^{\prime}\right|^{2 q} d s \leqslant R$ следует, что $\int_{0}^{\tau}\left|g^{\prime}\right|^{2 q} d s \leqslant R$.

Предположим, что выполнено следующее условие.

(m) Функция $\lambda_{0} \in K_{0}$.

Теорема 4. Пусть выполнены условия (c), (e), (g)-(i), (l), (m), а также следуюиее условие:

(n) 1) $\quad \alpha_{1} \in C^{2}\left([0, \tau] \times\left[\lambda_{*}, \lambda^{*}\right]\right)$;

2) $\quad \alpha_{2,3} \in C^{2}\left(\mathbf{R}^{+} \times\left[\lambda_{*}, \lambda^{*}\right]\right)$; 
3) $n р и i=2,3\left|\partial^{2} \alpha_{i}(s, x) / \partial s \partial x\right| \leqslant D_{s}$, причем $D_{s}$ ограничена $u$ $\overline{\lim }_{T \rightarrow \infty}(\sqrt{T})^{-1} \int_{0}^{T} s D_{s}^{2} d s<\infty$;

4) $\left|\frac{\partial^{2} \alpha_{1}(s, x)}{\partial s \partial x}-\frac{\partial^{2} \alpha_{1}(s, y)}{\partial s \partial x}\right|+\left|\frac{\partial^{2} \alpha_{1}(s, x)}{\partial x^{2}}-\frac{\partial^{2} \alpha_{1}(s, y)}{\partial x^{2}}\right| \leqslant C|x-y|$,

5) $\quad\left|\frac{\partial^{2} \alpha_{i}(s, x)}{\partial s \partial x}-\frac{\partial^{2} \alpha_{i}(s, y)}{\partial s \partial x}\right| \leqslant D_{s}|x-y|, \quad i=2,3$,

где $D_{s}$ - величины из условия 3 );

6) прu $i=2,3$

$$
\sup _{0 \leqslant s \leqslant \tau, \lambda * \leqslant x \leqslant \lambda^{*}} \frac{1}{n} \sum_{k=0}^{n-1}\left|\frac{\partial \alpha_{i}(s+k \tau, x)}{\partial x}\right|^{2}=O\left(\frac{1}{n^{1 / 4}}\right) .
$$

7) при $i=2,3\left|\partial^{2} \alpha_{1}(s, x) / \partial x^{2}\right| \leqslant F_{s}$, причем $F_{s}$ ограничена $и$ $\varlimsup_{T \rightarrow \infty} T^{-1} \int_{0}^{T}\left(s F_{s}^{2}\right)^{P} d s<\infty$

8)

$$
\left|\frac{\partial^{2} \alpha_{i}(s, x)}{\partial x^{2}}-\frac{\partial^{2} \alpha_{i}(s, y)}{\partial x^{2}}\right| \leqslant G_{s}|x-y|, \quad i=2,3,
$$

где $G_{s}$ ограничена $u \varlimsup_{T \rightarrow \infty} T^{-1 /(q+1)} \int_{0}^{T}\left(s G_{s}^{2}\right)^{q /(q+1)} d s<\infty$.

Тогда для любой бункиии $\varphi \in \bar{W}_{2 q}^{1}$ при $T \rightarrow \infty$ распределение случайной величины

$$
\sqrt{\frac{T}{\tau}} \int_{0}^{\tau} \varphi(t)\left(\lambda_{T}(t)-\lambda_{0}(t)\right) d t
$$

слабо сходится $к$ нормальному распределению с нулевым средним и дисперсией, равной

$$
\int_{0}^{\tau} \varphi^{2}(t)\left(\frac{1}{\lambda_{0}(t)}+\left|\frac{\partial \alpha_{1}\left(t, \lambda_{0}\right)}{\partial x}\right|^{2}\right)^{-1} d t
$$

Д о к а 3 а т е л ь с т в о. По условию $(\mathrm{m})$ сушествует такое $\varepsilon>0$, что $\lambda_{0}(t) \geqslant \varepsilon, t \in[0, \tau]$. В силу теоремы 2 и теоремы вложения соболевских пространств $\lim _{T \rightarrow \infty} \mathbf{P}\left\{\lambda_{T}(t) \geqslant \varepsilon / 2, t \in[0, \tau]\right\}=1$. Далее рассматриваются только те $T$ и $\omega$, для которых последнее неравенство выполнено. Возьмем производную по Гато $\left\langle Q_{T}^{\prime}\left(\lambda_{T}\right), h\right\rangle$ от функционала $Q_{T}$ в точке $\lambda_{T}$ по направлению $h \in \bar{W}_{2}^{1}[0, \tau]$, причем $h$ продолжена периодически с периодом $\tau$ :

$$
\begin{aligned}
\left\langle Q_{T}^{\prime}\left(\lambda_{T}\right), h\right\rangle= & \frac{n \tau}{T} \frac{1}{\tau}\left[-\int_{0}^{\tau} h d s+\int_{0}^{\tau} \frac{\lambda_{0}}{\lambda_{T}} h d s+\int_{0}^{\tau} \frac{h}{\lambda_{T}} d A_{n}\right] \\
& -\frac{1}{T} \int_{0}^{T} \frac{\partial \alpha\left(s, \lambda_{T}\right)}{\partial x}\left[\alpha\left(s, \lambda_{T}\right)-\alpha\left(s, \lambda_{0}\right)\right] h(s) d s \\
& +\frac{1}{T} \int_{0}^{T} \frac{\partial \alpha\left(s, \lambda_{T}\right)}{\partial x} h(s) d w_{s}+\left\langle R_{T}^{\prime}\left(\lambda_{T}\right), h\right\rangle
\end{aligned}
$$


Гце

$$
\left\langle R_{T}^{\prime}\left(\lambda_{T}\right), h\right\rangle=\frac{1}{T}\left(-\int_{0}^{T-n \tau} h d s+\int_{0}^{T-n \tau} \frac{h}{\lambda_{T}} d N_{n+1}\right) .
$$

Напомним, что $A_{n}$ и $N_{n}$ введены в п. 4 .

Зафиксируем функцию $\psi \in \bar{W}_{2 q}^{1}$. По условию $(\mathrm{m})$ для некоторого $\delta>$ 0 функции $\lambda_{0} \pm \delta \psi$ принадлежат $K_{0}$. В силу выпуклости $K$ корректно определены две функции $q_{ \pm}(t):=Q_{T}\left(\lambda_{T}+t\left(\lambda_{0}-\lambda_{T} \pm \delta \psi\right)\right), t \in[0,1]$. Они достигают максимума при $t=0$, откуда

$$
q_{ \pm}^{\prime}(0):=\left\langle Q_{T}^{\prime}\left(\lambda_{T}\right), \lambda_{0}-\lambda_{T} \pm \delta \psi\right\rangle \leqslant 0
$$

Или

$$
\left|\left\langle Q_{T}^{\prime}\left(\lambda_{T}\right), \psi\right\rangle\right| \leqslant \frac{1}{\delta}\left\langle Q_{T}^{\prime}\left(\lambda_{T}\right), \Delta_{T}\right\rangle
$$

где $\Delta_{T}$ введено в п. 4.

Оценим правую часть (31). В статье [3] доказано, что при выполнении условий настояпей теоремы

$$
\begin{aligned}
\frac{n \tau}{T} \frac{1}{\tau}\left[-\int_{0}^{\tau} \Delta_{T} d s+\int_{0}^{\tau} \frac{\lambda_{0} \Delta_{T}}{\lambda_{T}} d s+\int_{0}^{\tau} \frac{\Delta_{T}}{\lambda_{T}} d A_{n}\right] & =\frac{o_{p}(1)}{\sqrt{T}} \\
\left\langle R_{T}^{\prime}\left(\lambda_{T}\right), \Delta_{T}\right\rangle & =\frac{o_{p}(1)}{T} .
\end{aligned}
$$

Оценим интеграл $I_{1}:=T^{-1} \int_{0}^{T}\left|\alpha_{x}^{\prime}\left(s, \lambda_{T}\right)\right|\left|\alpha\left(s, \lambda_{T}\right)-\alpha\left(s, \lambda_{0}\right)\right|\left|\Delta_{T}\right| d s$. Воспользуемся условием (i) и теоремой 3 :

$$
\begin{aligned}
I_{1} \leqslant & \frac{C}{T} \int_{0}^{T}\left|\alpha_{x}^{\prime}\left(s, \lambda_{T}\right)\right|\left|\Delta_{T}\right|^{2} d s \leqslant \frac{C(n+1)}{T} \int_{0}^{\tau}\left|\frac{\partial \alpha_{1}}{\partial x}\left(s, \lambda_{T}\right)\right|\left|\Delta_{T}\right|^{2} d s \\
& +\frac{C}{T} \int_{0}^{T}\left|\frac{\partial\left(\alpha_{2}+\alpha_{3}\right)}{\partial x}\left(s, \lambda_{T}\right)\right|\left|\Delta_{T}\right|^{2} d s \leqslant C_{1} \int_{0}^{\tau}\left|\Delta_{T}\right|^{2} d s=\frac{o_{p}(1)}{\sqrt{T}} .
\end{aligned}
$$

Обозначим производную $\Delta_{T}^{\prime}:=\lambda_{T}^{\prime}-\lambda_{0}^{\prime}$. Используя условия $\left.\left.(\mathrm{n}), 1\right), 2\right)$, и обозначение $B_{n}$ из п. 3 , оценим интеграл

$$
\begin{aligned}
I_{2}:= & \frac{1}{T}\left|\int_{0}^{T} \alpha_{x}^{\prime}\left(s, \lambda_{T}\right) \Delta_{T}(s) d w_{s}\right| \leqslant \frac{1}{T}\left|\int_{0}^{n \tau} \alpha_{x}^{\prime}\left(s, \lambda_{T}\right) \Delta_{T} d w_{s}\right| \\
& +\frac{1}{T}\left|\int_{n \tau}^{T} \alpha_{x}^{\prime}\left(s, \lambda_{T}\right) \Delta_{T} d w_{s}\right| \leqslant \frac{C}{n \tau}\left|\int_{0}^{\tau} \frac{\partial \alpha_{1}}{\partial x}\left(s, \lambda_{T}\right) \Delta_{T} d B_{n}\right| \\
& +\frac{1}{T}\left|\int_{0}^{n \tau} \frac{\partial\left(\alpha_{2}+\alpha_{3}\right)}{\partial x}\left(s, \lambda_{T}\right) \Delta_{T} d w_{s}\right|+\frac{1}{T}\left|\int_{n \tau}^{T} \alpha_{x}^{\prime}\left(s, \lambda_{T}\right) d w_{s}\right| \\
\leqslant & \frac{C\left|B_{n}(\tau)\right|}{n \tau}\left|\frac{\partial \alpha_{1}\left(\tau, \lambda_{T}(\tau)\right)}{\partial x} \Delta_{T}(\tau)\right| \\
& +\frac{C}{n \tau} \int_{0}^{\tau}\left|B_{n}(s)\right| \frac{d}{d s} \frac{\partial \alpha_{1}\left(s, \lambda_{T}(s)\right)}{\partial x} \mid d s \\
& +\frac{C}{n \tau} \int_{0}^{\tau}\left|B_{n}\right| \frac{\partial \alpha_{1}}{\partial x}\left(s, \lambda_{T}\right) \Delta_{T}^{\prime} \mid d s
\end{aligned}
$$




$$
\begin{aligned}
& +\frac{1}{T}\left|\frac{\partial\left(\alpha_{2}+\alpha_{3}\right)}{\partial x}\left(n \tau, \lambda_{T}(\tau)\right) \Delta_{T}(\tau)\right||w(n \tau)| \\
& +\frac{1}{T} \int_{0}^{n \tau}\left|\frac{d}{d s} \frac{\partial\left(\alpha_{2}+\alpha_{3}\right)}{\partial x}\left(s, \lambda_{T}\right)\right|\left|w_{s}\right| d s \\
& +\frac{1}{T} \int_{0}^{n \tau}\left|\frac{\partial\left(\alpha_{2}+\alpha_{3}\right)}{\partial x}\left(s, \lambda_{T}\right) \Delta_{T}^{\prime}\right|\left|w_{s}\right| d s \\
& +\frac{1}{T}\left|\int_{n \tau}^{T} \frac{\partial \alpha}{\partial x}\left(s, \lambda_{T}\right) d w_{s}\right| .
\end{aligned}
$$

Далее,

$$
\begin{aligned}
& \frac{d}{d s} \frac{\partial \alpha_{i}\left(s, \lambda_{T}\right)}{d x}=\frac{\partial^{2} \alpha_{i}\left(s, \lambda_{T}\right)}{d s d x}+\frac{\partial^{2} \alpha_{i}\left(s, \lambda_{T}\right)}{d x^{2}} ; \\
& \frac{\left|B_{n}(\tau)\right|}{n \tau}\left|\frac{\partial \alpha_{1}}{\partial x}\left(\tau, \lambda_{T}(\tau)\right) \Delta_{T}(\tau)\right|=\frac{o_{p}(1)}{\sqrt{T}} ; \\
& \frac{1}{n \tau} \int_{0}^{\tau}\left|B_{n}\right|\left|\frac{\partial^{2} \alpha_{1}\left(s, \lambda_{T}\right)}{\partial s \partial x} \Delta_{T}\right|_{d s} \\
& \leqslant\left.\frac{C}{\sqrt{n}}|| \frac{1}{\sqrt{n}} \sum_{i=0}^{n-1} Y_{i}\right|_{L_{2}[0, \tau]}\left(\int_{0}^{\tau}\left|\Delta_{T}\right|^{2} d s\right)^{1 / 2}=\frac{o_{p}(1)}{\sqrt{T}} ; \\
& \frac{1}{n \tau} \int_{0}^{\tau}\left|B_{n}\right|\left|\frac{\partial \alpha_{1}\left(s, \lambda_{T}\right)}{\partial x} \Delta_{t}^{\prime}\right| d s \\
& \leqslant \frac{C}{\sqrt{n}}\left\|\frac{1}{\sqrt{n}} \sum_{i=0}^{n-1} Y_{i}\right\|_{L_{2}[0, \tau]}\left(\int_{0}^{\tau}\left|\Delta_{T}^{\prime}\right|^{2} d s\right)^{1 / 2}=\frac{o_{p}(1)}{\sqrt{T}} ; \\
& \frac{1}{T}|w(n \tau)| \frac{\partial\left(\alpha_{2}+\alpha_{3}\right)}{\partial x}\left(n \tau, \lambda_{T}(\tau)\right) \Delta_{t}(\tau) \mid=\frac{o_{p}(1)}{\sqrt{T}} .
\end{aligned}
$$

По условию $(\mathrm{n}), 3$ ) и по теореме 3

$$
\begin{aligned}
\frac{1}{T} & \int_{0}^{n \tau}\left|\frac{\partial^{2}\left(\alpha_{2}+\alpha_{3}\right)}{\partial s \partial x}\left(s, \lambda_{T}\right) \Delta_{T}\right|\left|w_{s}\right| d s \\
& \leqslant \frac{1}{T}\left(\int_{0}^{n \tau} D_{s}^{2} w_{s}^{2} d s\right)^{1 / 2} \sqrt{n}\left(\int_{0}^{\tau}\left|\Delta_{T}\right|^{2} d s\right)^{1 / 2} \\
& \leqslant\left(\frac{1}{\sqrt{T}} \int_{0}^{T} D_{s}^{2} w_{s}^{2} d s\right)^{1 / 2} \frac{o_{p}(1)}{\sqrt{T}}=\frac{o_{p}(1)}{\sqrt{T}}
\end{aligned}
$$

поскольку $\overline{\lim }_{T \rightarrow \infty}(\sqrt{T})^{-1} \mathrm{E} \int_{0}^{T} D_{s}^{2} w_{s}^{2} d s=\overline{\lim }_{T \rightarrow \infty}(\sqrt{T})^{-1} \int_{0}^{T} D_{s}^{2} s d s<$ $\infty$. По условию $(1), 2$ ) имеем:

$$
\begin{aligned}
& \frac{1}{T} \int_{0}^{n \tau}\left|\frac{\partial\left(\alpha_{2}+\alpha_{3}\right)}{\partial x} \Delta_{T}^{\prime}\right|\left|w_{s}\right| d s \\
& \quad \leqslant \sqrt{\frac{n}{T}}\left(\int_{0}^{\tau}\left|\Delta_{T}^{\prime}\right|^{2} d s\right)^{1 / 2} \frac{1}{\sqrt{T}}\left(\int_{0}^{n \tau} C_{s}^{2} w_{s}^{2} d s\right)^{1 / 2}=\frac{o_{p}(1)}{\sqrt{T}}
\end{aligned}
$$


«Остаток» в правой части (32) оценивается аналогично (33), следовательно, $I_{2}=o_{p}(1) / \sqrt{T}$. Итак, $\left|\left\langle Q_{T}^{\prime}\left(\lambda_{T}\right), \psi\right\rangle\right|=o_{p}(1) / \sqrt{T}$.

Подставим в $(30)$ функцию $\psi$ вместо $h$. В силу теоремы 3 из работы [3]

$$
\begin{aligned}
\left\langle Q_{T}^{\prime}\left(\lambda_{T}\right), \psi\right\rangle= & -\frac{1}{T} \int_{0}^{T} \alpha_{x}^{\prime}\left(s, \lambda_{T}\right)\left[\alpha\left(s, \lambda_{T}\right)-\alpha\left(s, \lambda_{0}\right)\right] \psi d s \\
& +\frac{1}{T} \int_{0}^{T} \alpha_{x}^{\prime}\left(s, \lambda_{T}\right) \psi d w_{s}+\frac{1+o(1)}{\tau} \\
& \times\left(-\int_{0}^{\tau} \frac{\Delta_{T} \psi}{\lambda_{0}} d s+\int_{0}^{\tau} \frac{\psi}{\lambda_{0}} d A_{n}\right)+\frac{o_{p}(1)}{\sqrt{T}}
\end{aligned}
$$

Оценим интеграл

$$
\begin{aligned}
& \frac{1}{T} \int_{0}^{T} \alpha_{x}^{\prime}\left(s, \lambda_{T}\right) \psi d w_{s}=\frac{1}{T} \int_{0}^{T} \alpha_{x}^{\prime}\left(s, \lambda_{0}\right) \psi d w_{s} \\
& \quad+\frac{1}{T} \int_{0}^{T}\left[\alpha_{x}^{\prime}\left(s, \lambda_{T}\right)-\alpha_{x}^{\prime}\left(s, \lambda_{0}\right)\right] \psi d w_{s}:=J_{1}+J_{2}
\end{aligned}
$$

Далее полагаем $T=n \tau ;$ при $n \tau \leqslant T<(n+1) \tau$ проводятся соответствующие оценки «остатков». Тогда

$$
J_{1}=\frac{1}{n \tau} \int_{0}^{\tau} \frac{\partial \alpha_{1}}{\partial x}\left(s, \lambda_{0}\right) \psi d B_{n}+\frac{1}{n \tau} \int_{0}^{n \tau} \frac{\partial\left(\alpha_{2}+\alpha_{3}\right)}{\partial x}\left(s, \lambda_{0}\right) \psi d w_{s}
$$

По центральной предельной теореме при $n \rightarrow \infty$

$$
\frac{1}{\tau \sqrt{n}} \int_{0}^{\tau} \frac{\partial \alpha_{1}}{\partial x}\left(s, \lambda_{0}\right) \psi d B_{n} \Longrightarrow \mathcal{N}\left(0, \frac{1}{\tau^{2}} \int_{0}^{\tau}\left|\frac{\partial \alpha_{1}}{\partial s}\left(s, \lambda_{0}\right)\right|^{2} \psi^{2} d s\right)
$$

по распределению. Далее, по условию (n), (6)

$$
\begin{aligned}
& \mathbf{E}\left|\frac{1}{\tau \sqrt{n}} \int_{0}^{n \tau} \frac{\partial\left(\alpha_{2}+\alpha_{3}\right)}{\partial x}\left(s, \lambda_{0}\right) \psi d w_{s}\right|^{2} \\
& \quad \leqslant \frac{C_{1}}{n} \int_{0}^{\tau} \psi^{2} \sum_{k=0}^{n-1}\left|\frac{\partial\left(\alpha_{2}+\alpha_{3}\right)\left(s+k \tau, \lambda_{0}(s)\right)}{\partial x}\right|^{2} d s=o(1), \quad n \rightarrow \infty .
\end{aligned}
$$

Итак,

$$
J_{1} \sqrt{n} \Longrightarrow \mathcal{N}\left(0, \frac{1}{\tau^{2}} \int_{0}^{\tau}\left|\frac{\partial \alpha_{1}}{\partial s}\left(s, \lambda_{0}\right)\right|^{2} \psi^{2} d s\right)
$$

по распределению. Оценим $J_{2}$ :

$$
\begin{aligned}
J_{2} \leqslant & \frac{1}{n \tau}\left|\int_{0}^{\tau}\left(\frac{\partial \alpha_{1}}{\partial x}\left(s, \lambda_{T}\right)-\frac{\partial \alpha_{1}}{\partial x}\left(s, \lambda_{0}\right)\right) \psi d B_{n}\right| \\
& +\frac{1}{n \tau}\left|\int_{0}^{n \tau}\left(\frac{\partial\left(\alpha_{2}+\alpha_{3}\right)}{\partial x}\left(s, \lambda_{T}\right)-\frac{\partial\left(\alpha_{2}+\alpha_{3}\right)}{\partial x}\left(s, \lambda_{0}\right)\right) \psi d w_{s}\right|
\end{aligned}
$$




$$
\begin{aligned}
\leqslant & \frac{C\left|\lambda_{T}(\tau)-\lambda_{0}(\tau)\right|}{n \tau}|\psi(\tau)|\left|B_{n}(\tau)\right| \\
& +\frac{1}{n \tau}\left(\int_{0}^{\tau}\left|\frac{d}{d s}\left(\frac{\partial \alpha_{1}}{\partial x}\left(s, \lambda_{T}\right)-\frac{\partial \alpha_{1}}{\partial x}\left(s, \lambda_{0}\right)\right)\right|^{2} \psi^{2} d s\right)^{1 / 2}\left\|\sum_{i=0}^{n-1} Y_{i}\right\|_{L_{2}[0, \tau]} \\
& +\left\|\sum_{i=0}^{n-1} Y_{i}\right\|_{L_{2}[0, \tau]} \frac{1}{n \tau}\left(\int_{0}^{\tau}\left|\psi^{\prime}\right|^{2}\left|\frac{\partial \alpha_{1}}{\partial x}\left(s, \lambda_{T}\right)-\frac{\partial \alpha_{1}}{\partial x}\left(s, \lambda_{0}\right)\right|^{2} d s\right)^{1 / 2} \\
& +\frac{C|w(n \tau)|}{n \tau}|\psi(T)| \frac{\partial\left(\alpha_{2}+\alpha_{3}\right)}{\partial x}\left(T, \lambda_{T}(T)\right)-\frac{\partial\left(\alpha_{2}+\alpha_{3}\right)}{\partial x}\left(T, \lambda_{0}(T)\right) \mid \\
& +\frac{1}{n \tau} \int_{0}^{n \tau}\left|\frac{\partial^{2}\left(\alpha_{2}+\alpha_{3}\right)}{\partial s \partial x}\left(s, \lambda_{T}\right)-\frac{\partial^{2}\left(\alpha_{2}+\alpha_{3}\right)}{\partial s \partial x}\left(s, \lambda_{0}\right)\right||\psi|\left|w_{s}\right| d s \\
& +\frac{1}{n \tau} \int_{0}^{n \tau}\left|\frac{\partial\left(\alpha_{2}+\alpha_{3}\right)}{\partial x}\left(s, \lambda_{T}\right)-\frac{\partial\left(\alpha_{2}+\alpha_{3}\right)}{\partial x}\left(s, \lambda_{0}\right)\right|\left|\psi^{\prime}\right|\left|w_{s}\right| d s \\
\leqslant & \frac{C}{\sqrt{n}}\left\|\frac{1}{\sqrt{n}} \sum_{i=0}^{n-1} Y_{i}\right\|\left\|_{L_{2}[0, \tau]}\left(\int_{0}^{\tau}\left|\psi^{\prime}\right|^{2 q} d s\right)^{1 /(2 q)}\right\| \lambda_{T}-\lambda_{0} \|_{W_{2 p}^{1}}^{1} \\
& +\frac{1}{n \tau} \int_{0}^{n \tau} D_{s}\left|\Delta_{T}\right||\psi|\left|w_{s}\right| d s \\
& +\frac{1}{n \tau} \int_{0}^{n \tau} C_{s}\left|\Delta_{T}\right|\left|\psi^{\prime}\right|\left|w_{s}\right| d s+\frac{o_{p}(1)}{\sqrt{n}}=\frac{o_{p}(1)}{\sqrt{n}} .
\end{aligned}
$$

Оценим интеграл

$$
J_{3}:=T^{-1} \int_{0}^{T} \alpha_{x}^{\prime}\left(s, \lambda_{T}\right)\left[\alpha\left(s, \lambda_{T}\right)-\alpha\left(s, \lambda_{0}\right)\right] \psi d s .
$$

Имеем:

$$
J_{3}=\frac{1}{\tau} \int_{0}^{\tau} \frac{1}{n} \sum_{i=0}^{n-1}\left|\alpha_{x}^{\prime}\left(s+i \tau, \lambda_{0}\right)\right|^{2} \Delta_{T} \psi d s+\frac{o_{p}(1)}{\sqrt{n}} .
$$

Из условия (n), 6) и теоремы 3 получаем:

$$
J_{3}=\frac{1}{\tau} \int_{0}^{\tau}\left|\frac{\partial \alpha_{1}}{\partial x}\left(s, \lambda_{0}\right)\right|^{2} \Delta_{T} \psi d s+r_{n}+\frac{o_{p}(1)}{\sqrt{n}},
$$

где

$$
\begin{aligned}
\left|r_{n}\right| & \leqslant \frac{C}{T^{1 / 4}} \int_{0}^{\tau}\left|\Delta_{T}\right||\psi| d s \\
& \leqslant \frac{C}{\sqrt{T}}\left(\int_{0}^{\tau} \psi^{2} d s \cdot T^{1 / 2} \cdot \int_{0}^{\tau}\left|\Delta_{T}\right|^{2} d s\right)^{1 / 2}=\frac{o_{p}(1)}{\sqrt{T}} .
\end{aligned}
$$

Из (34), (31) следует представление:

$$
\sqrt{n} \tau J_{3}+\sqrt{n} \int_{0}^{\tau} \frac{\Delta_{T} \psi}{\lambda_{0}} d s=\tau J_{1} \sqrt{n}+\sqrt{n} \int_{0}^{\tau} \frac{\psi}{\lambda_{0}} d A_{n}+o_{p}(1) .
$$


Отсюда

$$
\begin{aligned}
& \sqrt{n} \int_{0}^{\tau}\left(\frac{1}{\lambda_{0}}+\left|\frac{\partial \alpha_{1}\left(s, \lambda_{0}\right)}{\partial x}\right|^{2}\right) \Delta_{T} \psi d s \\
& \Longrightarrow \mathcal{N}\left(0, \int_{0}^{\tau}\left(\frac{1}{\lambda_{0}}+\left|\frac{\partial \alpha_{1}\left(s, \lambda_{0}\right)}{\partial x}\right|^{2}\right) \psi^{2} d s\right.
\end{aligned}
$$

при $n \rightarrow \infty$ по распределению. Полагая здесь

$$
\varphi:=\left(\frac{1}{\lambda_{0}}+\left|\frac{\partial \alpha_{1}\left(s, \lambda_{0}\right)}{\partial x}\right|^{2}\right) \psi
$$

получаем искомое соотношение:

$$
\sqrt{\frac{T}{\tau}} \int_{0}^{\tau} \varphi \Delta_{T} d s \Longrightarrow \mathcal{N}\left(0, \int_{0}^{\tau} \varphi^{2}\left(\frac{1}{\lambda_{0}}+\left|\frac{\partial \alpha_{1}\left(s, \lambda_{0}\right)}{\partial x}\right|^{2}\right)^{-1} d s\right)
$$

Теорема 4 доказана.

6. Сравнение оценок. Показателем эффективности рассмотренной оценки является асимптотическая дисперсия гладкого функционала от неe:

$$
\sigma^{2}(\varphi):=\int_{0}^{\tau} \varphi^{2}\left(\frac{1}{\lambda_{0}}+\left|\frac{\partial \alpha_{1}\left(s, \lambda_{0}\right)}{\partial x}\right|^{2}\right)^{-1} d s
$$

Если строить ОМП $\tilde{\lambda}_{T}$ по пуассоновской компоненте $\left\{N_{t}\left(\lambda_{0}\right), 0 \leqslant t \leqslant T\right\}$, то, согласно [3],

$$
\sqrt{\frac{T}{\tau}} \int_{0}^{\tau} \varphi\left(\tilde{\lambda}_{T}-\lambda_{0}\right) d s \Longrightarrow \mathcal{N}\left(0, \int_{0}^{\tau} \varphi^{2} \lambda_{0} d s\right)
$$

и асимптотическая дисперсия равна: $\tilde{\sigma}^{2}(\varphi):=\int_{0}^{\tau} \varphi^{2} \lambda_{0} d s$. Если же рассматривать ОМП $\hat{\lambda}_{T}$ по наблюдениям $\left\{\eta_{t}\left(\lambda_{0}\right), 0 \leqslant t \leqslant T\right\}$, то при условии

$$
\frac{\partial \alpha_{1}(s, x)}{\partial s} \neq 0, \quad s \in[0, \tau], x \in\left[\lambda_{*}, \lambda^{*}\right]
$$

будем иметь:

$$
\sqrt{\frac{T}{\tau}} \int_{0}^{\tau} \varphi\left(\hat{\lambda}_{T}-\lambda_{0}\right) d s \Longrightarrow \mathcal{N}\left(0, \int_{0}^{\tau} \varphi^{2}\left|\frac{\partial \alpha_{1}\left(s, \lambda_{0}\right)}{\partial x}\right|^{-2} d s\right)
$$

и асимптотическая дисперсия равна:

$$
\widehat{\sigma}^{2}(\varphi):=\int_{0}^{\tau} \varphi^{2}\left|\frac{\partial \alpha_{1}\left(s, \lambda_{0}\right)}{\partial x}\right|^{-2} d s
$$

В условиях теоремы 4 при $\varphi \in \bar{W}_{2 q}^{1}, \varphi \neq 0$ справедливо неравенство:

$$
\sigma^{2}(\varphi) \leqslant \widehat{\sigma}^{2}(\varphi)
$$


а при дополнительном условии $(35) \sigma^{2}(\varphi)<\tilde{\sigma}^{2}(\varphi)$. В соотношении (36) будет строгое неравенство, если

$$
\operatorname{mes}\left\{s \in \operatorname{supp} \varphi: \frac{\partial \alpha_{1}\left(s, \lambda_{0}(s)\right)}{\partial x} \neq 0\right\}>0 .
$$

Итак, совместная обработка наблюдаемых компонент $\left\{N_{t}\left(\lambda_{0}\right), \eta_{t}\left(\lambda_{0}\right)\right\}$ более эффективна, чем их раздельная обработка.

Авторы благодарят рецензента за устранение некоторых недостатков в изложении материала статьи.

\section{СПИСОК ЛИТЕРАТУРЫ}

1. Дороговчев А.Я. Теорня оценок параметров случайных процессов. Киев: Вища школа, 1982. $192 \mathrm{c.}$

2. Ибрамхалияов И. II., Скороход А.В. Состоятельные оценки параметров случайных процессов. Киев: Наукова думка, 1980. 189 с.

3. Дороговчев $A . Я$., Кукуи А.Г. Асимптотические свойства непараметрической оценки интенсивности неоднородного процесса Пуассона. - Кибернетика и системный анализ, 1996, № 1, с. 91-104.

4. Скороход А. В. Случайные процессы с независимыми приращениями. М.: Наука, 1964. $278 \mathrm{c.}$

5. Кабанов Ю. М., Липчер P. III., IIирдев А. Н. Мартингальные методы в теории точечных процессов. - Тр. школы-семинара по теории случайных процессов (Друскининкай, 1974), т. II, Вильнюс: ИФМ АН ЛитССР, 1975, с. 269-354.

6. Какторович Л. В., Акимов Г. П. Функциональный анализ. М.: Наука, 1984. $752 \mathrm{c}$.

7. Аркия В.И., Евстианеев И. В. Вероятностные модели управления и экономической динамики. М.: Наука, 1979. 202 с.

8. Dorogovtsev A. Ya., Zerek N., Kukush A.G. Weak convergence of an infinitedimensional parameter estimator to a normal distribution. - Theory Probab. and Math. Statist., 1988, № 37, p. 45-51.

9. Петров В.В. Суммы независимых случайных величин. М.: Наука, 1972. 416 с.

10. Лunчер P. III., ШІиряев A. Н. Статистика случайных процессов. М.: Наука, 1974. $696 \mathrm{c}$.

11. Revesz P. Die Gesetze der grossen Zahlen. Budapest: Acad. Kiado, 1968. 173 p.

12. Рисс $\Phi$., Секефальви-Надь Б. Лекции по функциональному анализу. М.: Мир, 1979. $587 \mathrm{c}$. 